\title{
Modelling the timing of Betula pubescens budburst. II. Integrating complex effects of photoperiod into process-based models
}

\author{
Amelia Caffarra $^{1, *}$, Alison Donnelly ${ }^{2}$, Isabelle Chuine ${ }^{3}$ \\ ${ }^{1}$ Research and Innovation Centre, Agriculture Area, Fondazione Edmund Mach, San Michele all'Adige, 38100 Trento, Italy \\ ${ }^{2}$ Department of Botany, School of Natural Sciences, Trinity College Dublin, Dublin 2, Ireland \\ ${ }^{3}$ CEFE-CNRS, 1919 route de Mende, 34293 Montpellier, France
}

\begin{abstract}
Despite experimental evidence of the individual and interactive effects of photoperiod and temperature on bud growth, photoperiod has not yet been effectively accounted for in models of budburst. However, in some tree species, such as Betula pubescens (birch), photoperiod has an important role in phenological control, and its inclusion in process-based models of budburst might affect phenological projections under climate change scenarios. The aim of the present study was to integrate photoperiod into a process-based phenological model (Chuine 2000; J Theor Biol 207: 337-347; Unified model), using experimental findings in which photoperiod was found to significantly affect budburst in B. pubescens (Caffarra et al. 2011; Clim Res 46:147-157, this issue). The effect of photoperiod was integrated into the model at 2 levels. Firstly, photoperiod, in interaction with temperature, affects the course of dormancy induction. Secondly, photoperiod modifies the response to temperature during the phase of forcing. The resulting model (DORMPHOT) for the simulation of birch budburst was fitted to a large phenological dataset, including data from different latitudes, and validated with 7 datasets from 4 different European countries. Besides giving more biological realism to the model, the newly introduced mechanisms improved its predictive performance. The DORMPHOT model outperformed the Unified model, the linear regression model (budburst date vs. spring average temperature), and the UniForc model. It also proved to be more accurate at predicting budburst in extremely warm years, which suggests it might be more reliable than previous models when using future climate change scenarios.
\end{abstract}

KEY WORDS: Betula pubescens - Budburst - Calibration - Phenological models - Photoperiod . Validation

Resale or republication not permitted without written consent of the publisher

\section{INTRODUCTION}

It is becoming increasingly important to understand and predict how tree growth responds to changes in environmental conditions and, in particular, to global warming. Year-to-year variations in tree phenology are indeed responsible for large differences in forest productivity and carbon sequestration (Kellomaki \& Kolstrom 1994, Lieth 1997, Leinonen \& Kramer 2002, Piao et al. 2007). In addition, earlier budburst due to global warming may lead to extensive damage from early frost episodes (Hänninen 1995, Kramer et al. 2000). Different models of tree phenology have been pro- posed to predict the timing of budburst and flowering of tree species by the input of environmental variables, such as temperature and daylength (see, for example, Landsberg 1974, Richardson et al. 1974, Sarvas 1974, Hänninen 1995, Chuine 2000, Donnelly et al. 2006).

As spring temperature is the most important factor affecting the timing of budburst, the simplest possible approach is to link budburst date and mean monthly temperatures using an empirical relationship (see, for example, Donnelly et al. 2006). On the other hand, a more sophisticated approach is possible, with the use of process-based modelling. Experimental studies have shown that the response of a dormant plant to 
temperature changes as dormancy progresses, and its responses are, in some cases, proportional to the amount of time of exposure to a particular environmental condition. These findings have resulted in the elaboration of models that describe budburst timing as the end point of 2 consecutive phases: endodormancy, which is released upon the cumulative effect of chilling temperatures (cool, autumn-winter temperatures), and ecodormancy, during which the cumulative effect of forcing temperatures (warm, spring temperatures) promotes cell growth (Hänninen 1990, Battey 2000). These models account for the effects of temperature in terms of developmental units, and different functions relate temperature to the rate of growth or the rate of dormancy release of the buds.

Among these models, the simplest consider only the effects of forcing temperatures for the prediction of budburst timing. In the Spring Warming model (Cannell \& Smith 1983, Chuine et al. 1998, Pop et al. 2000), also called the Thermal Time model (Hunter \& Lechowicz 1992), and in the UniForc model (Chuine 2000), temperature is linearly or sigmoidally related to the rate of growth of the developing bud, and developmental units are accumulated from a starting date until a critical threshold is reached and budburst occurs.

Other models also consider the effect of chilling temperatures for dormancy release, but differ as to the timing of the periods during which chilling and forcing temperatures are active. While some models consider ontogenetic development to start only after the fulfilment of a fixed chilling requirement, after which chilling has no further effect on bud growth (Richardson et al. 1974, Sarvas 1974, Hänninen 1990), others use a more dynamic representation of dormancy transition and do not consider dormancy as a sequence of steady states separated by boundaries (Landsberg 1974, Cannell \& Smith 1983). Chuine (2000) proposed the Unified model, in which the functions describing the above models were shown to be all particular cases of more general functions.

In addition to the effect of temperature, other environmental variables have an important role in determining budburst. Photoperiod has been shown to control the timing of phenological events of a number of tree species, including Betula pubescens (birch) (Thomas \& Vince Prue 1997). For example, it is known that photoperiod is the main trigger of growth cessation for this species (Håbjørg 1972, Thomas \& Vince Prue 1997, Welling et al. 1997). Photoperiod also seems to affect growth resumption in partially chilled birch trees and interact with temperature (Heide 1993, Myking \& Heide 1995). However, at present, these effects are not realistically integrated into phenological models of budburst. Indeed, the effects of photoperiod on budburst timing have been previously modelled as: (1) a change in chilling rate through a multiplication coefficient $K$ (Campbell \& Sugano 1975), (2) an additive factor (Kramer 1994) and (3) through the use of a fixed calendar date as a starting point for bud onthogenesis (Häkkinen et al. 1998). However, these attempts have given conflicting results. For example, while Häkkinen et al. (1998) found that the use of photoperiod improved budburst predictions, Kramer (1994) noted that photosensitivity reduced the predictive power of models.

Caffarra et al. (2011, this issue) investigated the individual effects and interaction of temperature and photoperiod on the rates of dormancy induction and release in birch through a series of controlled environment experiments, in order to develop a conceptual model of dormancy for this species. The experiments were conducted on the Betula pubescens clone grown in the international phenological gardens (IPGs) network, which comprises 89 garden sites throughout Europe (www.agrar.hu-berlin.de/struktur/institute/nptw/ agrarmet/phaenologie/ipg; Chmielewski 1996). Their results showed that photoperiod affected the timing of budburst only if coupled with warm, forcing temperatures, suggesting that photoperiod affected forcing rate rather than chilling rate. Caffarra et al. (2011) summarised their findings into a general framework laying the foundation for the formalisation of a model explaining birch budburst in terms of temperature and photoperiod.

The aim of the present work is to integrate photoperiod into the temperature-driven, process-based Unified model of Chuine (2000), based on the experimental findings of Caffarra et al. (2011) and on previous studies (Heide 1993, Myking \& Heide 1995), in order to account for the experimentally observed responses to this environmental factor, and to increase the realism of budburst models for this species. This will help to refine our understanding of the functional relationships between environmental drivers and dormancy release and to lead to more accurate predictions of budburst timing, in particular when using climate change scenarios.

\section{MATERIALS AND METHODS}

\subsection{Phenological data}

The phenological series used to calibrate and test the model are from 8 IPG sites from 4 different European countries: Ireland, Germany, Switzerland and Norway. Their description is given in Table 1. One dataset (Valentia, Ireland) was used for parameterisation only, the remaining 7 were used for validation. The temperature data (mean daily air temperature) were obtained from nearby $(<24 \mathrm{~km})$ stations, belonging to the na- 
Table 1. Geographical position (latitude, longitude, altitude) of the International Phenological Garden (IPG) sites and the meteorological stations used in the present study; n: number of years with observations of budburst date

\begin{tabular}{|c|c|c|c|c|c|c|c|}
\hline \multirow[t]{2}{*}{ Site } & \multirow[t]{2}{*}{$\mathrm{n}$} & \multicolumn{3}{|c|}{ - IPG } & \multicolumn{3}{|c|}{ Meteorological stations } \\
\hline & & Altitude (m) & Latitude & Longitude & Altitude (m) & Latitude & Longitude \\
\hline Valentia, Ireland ${ }^{\mathrm{a}}$ & 24 & 14 & $51^{\circ} 56^{\prime} \mathrm{N}$ & $10^{\circ} 15^{\prime} \mathrm{W}$ & 14 & $51^{\circ} 56^{\prime} \mathrm{N}$ & $10^{\circ} 15^{\prime} \mathrm{W}$ \\
\hline Johnstown, Ireland $^{\mathrm{b}}$ & 10 & 60 & $52^{\circ} 18^{\prime} \mathrm{N}$ & $06^{\circ} 31^{\prime} \mathrm{W}$ & 60 & $52^{\circ} 18^{\prime} \mathrm{N}$ & $06^{\circ} 31^{\prime} \mathrm{W}$ \\
\hline JFK Arboretum, Ireland ${ }^{\mathrm{b}}$ & 22 & 80 & $52^{\circ} 20^{\prime} \mathrm{N}$ & $06^{\circ} 38^{\prime} \mathrm{W}$ & 80 & $52^{\circ} 20^{\prime} \mathrm{N}$ & $06^{\circ} 38^{\prime} \mathrm{W}$ \\
\hline Wulfsdorf, Germanyc & 24 & 46 & $53^{\circ} 39^{\prime} \mathrm{N}$ & $10^{\circ} 12^{\prime} \mathrm{E}$ & 43 & $53^{\circ} 40^{\prime} \mathrm{N}$ & $10^{\circ} 12^{\prime} \mathrm{E}$ \\
\hline Freiburg(-Eschbach), Germany ${ }^{\mathrm{c}}$ & 26 & 270 & $48^{\circ} 01^{\prime} \mathrm{N}$ & $07^{\circ} 59^{\prime} \mathrm{E}$ & 330 & $48^{\circ} 00^{\prime} \mathrm{N}$ & $7^{\circ} 51^{\prime} \mathrm{E}$ \\
\hline Munchen, Germany ${ }^{\mathrm{b}}$ & 17 & 540 & $48^{\circ} 11^{\prime} \mathrm{N}$ & $11^{\circ} 10^{\prime} \mathrm{E}$ & 530 & $48^{\circ} 13^{\prime} \mathrm{N}$ & $11^{\circ} 07^{\prime} \mathrm{E}$ \\
\hline Zurich, Switzerland $^{\mathrm{b}}$ & 9 & 600 & $47^{\circ} 21^{\prime} \mathrm{N}$ & $08^{\circ} 27^{\prime} \mathrm{E}$ & 450 & $47^{\circ} 28^{\prime} \mathrm{N}$ & $8^{\circ} 32^{\prime} \mathrm{E}$ \\
\hline Bergen, Norway ${ }^{\mathrm{c}}$ & 7 & 50 & $60^{\circ} 16^{\prime} \mathrm{N}$ & $05^{\circ} 21^{\prime} \mathrm{E}$ & 50 & $60^{\circ} 28^{\prime} \mathrm{N}$ & $5^{\circ} 23^{\prime} \mathrm{E}$ \\
\hline
\end{tabular}

tional meteorological service of each country (see Table 1 for their coordinates). Daylength $(D L)$ at each site was calculated as follows:

if $a>1$, then $D L(t)=0$

if $a<-1$, then $D L(t)=24$

if $-1 \leq a \leq 1$, then $D L(t)=24 / \pi \times \arccos (a)$

where $t$ is the day of year;

$$
\begin{aligned}
a=- & \tan (\text { lat. } \times \pi / 180) \times \\
& \tan \{-23.45 \pi / 180 \times \cos [2 \pi(t+10) / 365]\}
\end{aligned}
$$

where lat. is the latitude of the site in decimal degrees.

\subsection{Formalisation of the effects of photoperiod and temperature on the progress of dormancy}

We assumed that the timing of budburst depended on the rate of dormancy induction, the rate of chilling and the rate of forcing. These rates, described below, are functions of daily temperature and photoperiod. Their daily sums provide the states of dormancy induction, the state of dormancy (or chilling), of quiescence (or forcing) and, ultimately, the date of budburst, respectively.

\subsection{Dormancy induction}

Experimental evidence shows that dormancy induction in birch can be considered as a cumulative process, with dormancy being established after a critical number of dormancy induction units $\left(D_{\text {crit }}\right)$ have been received (Welling et al. 1997, Li et al. 2003), and that dormancy induction is promoted by low temperatures and a photoperiod below a critical threshold $\left(D L_{\text {crit }}\right)$.
Therefore, we hypothesised that the rate of dormancy induction was negatively related to both temperature and photoperiod through sigmoidal relationships and bound between 0 and 1 . Dormancy induction is thus completed at $t_{\mathrm{d}}$ when the state of dormancy induction $D S(t) \geq D_{\text {crit. }}$. Dormancy induction state $(D S)$ is defined by the daily accumulation of rates of dormancy triggered by temperature $\left(D R_{\mathrm{T}}\right)$ and photoperiod $\left(D R_{\mathrm{P}}\right)$ induction as follows:

with

$$
D S(t)=\sum_{t_{0}}^{t} D R_{\mathrm{T}}(t) \cdot D R_{\mathrm{P}}(t)
$$

$$
\begin{gathered}
D R_{\mathrm{T}}(t)=\frac{1}{1+\mathrm{e}^{a D\left(T_{t}-b D\right)}} \\
D R_{P}(t)=\frac{1}{1+\mathrm{e}^{10\left(D L_{t}-D L_{\text {crit }}\right)}}
\end{gathered}
$$

where $t_{0}$ is the starting date of dormancy induction (set as 1 September of the year preceding budburst), $T_{t}$ is the mean daily temperature on day $t\left({ }^{\circ} \mathrm{C}\right), D L_{t}$ is the daylength (hours) on day $t$ and $a D, b D$ and $D L_{\text {crit }}$ are function parameters. These functions are general enough to describe a wide range of responses to temperatures and photoperiods and can thus be applied to different species. The promoting effect of low temperatures in conjunction with short photoperiods during dormancy induction (Caffarra et al. 2011) was assumed to be due to a multiplicative relationship between the 2 rates of dormancy (Eq. 5).

\subsection{Chilling}

The rate of chilling was described by a modified version of the chilling function of the Unified model (Chuine 2000), which relates temperature to chilling rate using symmetric unimodal curves, with either a maximum or a minimum depending on the parameter estimates. As the response of endodormant trees to 
chilling temperatures is known to show a maximum, we set parameter $b C$ of Eq. (13) to 1 . The resulting simplified function has only 2 parameters and is flexible enough to fit bell-shaped chilling rates with a maximum and a gentle slope. This is in accordance with the shape of the function fitted by Sarvas (1974) to data regarding the effect of chilling temperature on dormancy release in birch seedlings. Thus, the state of chilling $(C S)$ was defined by the daily accumulation of rates of chilling $(C R)$ as follows:

$$
C S(t)=\sum_{t_{\mathrm{d}}}^{t} \frac{1}{1+\mathrm{e}^{a C\left(T_{t}-C C\right)^{2}+\left(T_{t}-c C\right)}}
$$

where $t_{\mathrm{d}}$ is the starting date of dormancy release (i.e. the day after the attainment of $D_{\text {crit }}$ ) and $a C$ and $c C$ are function parameters.

\subsection{Forcing}

The experiments conducted by Caffarra et al. (2011) confirmed the previously shown positive and sigmoidal relationship between temperature and the rate of forcing (Sarvas 1972, Hänninen 1990). Thus, the state of forcing $(F S)$ was defined by the daily accumulation of rates of forcing $F R(t)$ as follows:

$$
F S(t)=\sum_{t_{\mathrm{d}}}^{t} \frac{1}{1+\mathrm{e}^{d F\left(T_{t}-T_{50}\right)}}
$$

where $d F$ is a function parameter, which sets the positive slope of the curve $(d F \leq 0)$. The time of budburst was defined as the day when $F S \geq F_{\text {crit }}$.

According to the experimental results of Caffarra et al. (2011), the rate of forcing was affected by both photoperiod and the duration of the previous chilling exposure. Forcing photoperiod above a threshold promoted the rate of forcing (photoperiod sensitivity). This threshold was not constant, but decreased after increasing chilling durations from a maximum of 12-14 h to a minimum of $<10 \mathrm{~h}$ (effect of chilling on photoperiod sensitivity). Thus, long days increased forcing rate, but this effect was, in turn, affected by the duration of chilling exposure, with longer exposures resulting in a larger effect of long days.

To describe photoperiod sensitivity, the temperature of mid-response, $T_{50}$, was considered to depend on daylength, with longer daylength causing the forcing function, $F R(t)$, to shift towards lower temperatures, i.e. with a lower temperature of mid-response. Thus, increasing daylength resulted in an increase in the forcing rate elicited by a given temperature, without altering its optimum. The parameter $T_{50}$ was thus related to daylength by a sigmoidal decreasing function (longer photoperiods set a lower $T_{50}$ ) bound between $0^{\circ} \mathrm{C}$ (maximum growth competence, all temperatures $>0^{\circ} \mathrm{C}$ promote development) and $60^{\circ} \mathrm{C}$ (minimum growth competence, no forcing units are accumulated at ambient temperatures) as follows:

$$
T_{50}(t)=\frac{60}{1+\mathrm{e}^{g T\left(D L_{t}-D L_{50}(t)\right)}}
$$

The parameter $g T$ sets the negative slope of the curve $(g T \geq 0)$, and $D L_{50}(t)$ corresponds to the critical daylength at which the temperature of mid-response of the rate of forcing $(F R)$ is $30^{\circ} \mathrm{C}$. This parameter varies in time depending on the state of chilling following a decreasing sigmoidal function (high state of chilling set a lower $D L_{50}$ ) bound between 0 and $24 \mathrm{~h}$ as follows:

$$
D L_{50}(t)=\frac{24}{1+\mathrm{e}^{h D L\left(C S(t)-C_{\text {crit }}\right)}}
$$

where $C S(t)$ is the state of chilling on day $t$, and $h D L$ and $C_{\text {crit }}$ are 2 parameters to be fitted. The parameter $h D L$ sets the negative slope of the curve $(h D L \geq 0)$, and $C_{\text {crit }}$ corresponds to the critical state of chilling at which a daylength of $12 \mathrm{~h}$ sets a temperature of mid-response of $F R$ of $30^{\circ} \mathrm{C}$. Thus, $C_{\text {crit }}$ can be considered an indicator of the critical state of chilling needed for forcing temperatures to be active.

\subsection{The DORMPHOT model}

The DORMPHOT model (for DORMancy and PHOToperiod), in contrast to the Unified model, accounts for the process of dormancy induction by the effects of short photoperiods and low temperatures. In addition, its parameter $C_{\text {crit }}$ is not simply a threshold that determines a break in dormancy, but also that defines the form of the response function to photoperiod during the ecodormancy phase. Conversely, the parameter $F_{\text {crit }}$ is considered a constant in the DORMPHOT model rather than a dynamic threshold determined by the total amount of chilling accumulated over the season (Chuine 2000). Thus, the DORMPHOT model describes the response of cell growth to temperature during the ecodormancy phase as dynamic and dependent on the state of chilling and on photoperiod, unlike the Unified model, which describes the response as constant. This increase in complexity is accounted for by 2 additional parameters. Thus, the DORMPHOT model has 11 parameters: $D L_{\text {crit }}, D_{\text {crit }} C_{\text {crit }}, F_{\text {crit, }} a D, b D, a C, c C, d F, g T$ and $h D L$. A conceptual schematic representation of the DORMPHOT model is given in Fig. 1.

For further evaluation, we compared the accuracy of the DORMPHOT model with those of the Unified model (Chuine 2000), the UniForc model (Chuine et al. 1998) and a linear model (Donnelly et al. 2006). 


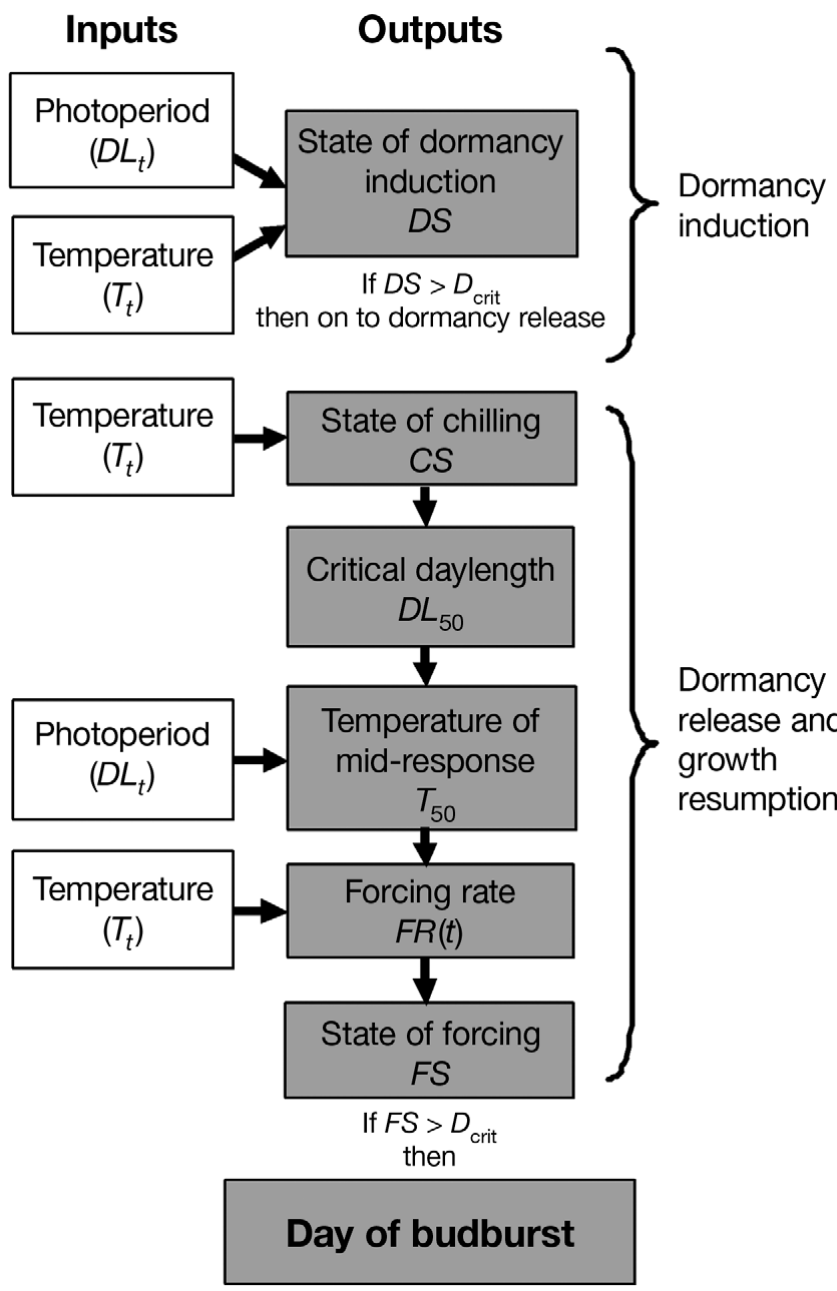

Fig. 1. Conceptual schema of the DORMPHOT model. White boxes: inputs required during the execution of the algorithm; grey boxes: outputs generated as a function of input variables and previous outputs during the different stages of the algorithm, and final output 'day of budburst'. For the specific functions making up the model, see Section 2.6

\subsection{Linear model}

The months February-April showed the best correlation with budburst date when compared to other winter-spring months at Irish IPG stations (Donnelly et al. 2006). The time of budburst $\left(t_{b}\right)$ is such that:

$$
t_{\mathrm{b}}=\mathrm{a}\left(T_{\mathrm{avg}}\right)+b
$$

where $T_{\text {avg }}$ stands for average temperature FebruaryApril and $a$ and $b$ are 2 parameters to be fitted.

\subsection{UNIFORC model}

The UniForc model was defined by 4 parameters, the starting date of forcing accumulation $\left(t_{0}\right)$, the 2 parameters defining the sigmoidal response curve to tem- perature $(d F, f F)$ and the critical state of forcing for budburst $\left(F_{\text {crit }}\right)$. The time of budburst was defined as the day $t$ when:

$$
F S(t)=\sum_{t_{0}}^{t} \frac{1}{1+\mathrm{e}^{d F\left(T_{t}-f F\right)}} \geq F_{\text {crit }}
$$

where $T_{t}$ stands for mean daily temperature.

\subsection{Unified model}

The Unified model has 9 parameters $f F, d F, a C, b C$, $c C, W, Z, t c, C_{\text {crit }}$ and defines the time of budburst as the day $t$ when the state of forcing $(F S)$ given by the daily accumulation of rates of forcing $F R(t)$ reaches $F_{\text {crit, }}$ such that:

$$
F S(t)=\sum_{t 1}^{t} \frac{1}{1+\mathrm{e}^{d F\left(T_{t}-f F\right)}} \geq F_{\text {crit }}
$$

where $t_{1}$ is the day $t$ when the state of chilling (CS) given by the daily accumulation of rates of chilling $C R(t)$ reaches $C_{\text {crit }}$ :

$$
t_{1} \text { such that } C S(t)=\sum_{t 0}^{t} \frac{1}{1+\mathrm{e}^{a C\left(T_{t}-c C\right)^{2}+b C\left(T_{t}-c C\right)}} \geq C_{\text {crit }}
$$

where $t_{0}$ is the starting date for chilling accumulation (set as 1 September of the year preceding budburst). The critical forcing requirement is calculated through the relationship:

$$
\begin{gathered}
F_{\text {crit }}=w \mathrm{e}^{z C S(t)} \quad \text { with } w>0 \text { and } z<0 \\
C S(t)=\sum_{t_{0}}^{t^{c}} C R(t)
\end{gathered}
$$

where $t c$ is the ending date for chilling accumulation.

The Unified model allows for a direct estimation of the response of bud growth to either chilling or forcing temperatures and the periods when these temperatures affect bud growth. It can be simplified according to standard statistical tests for any particular species; thus, its assumptions should not be constrained a priori (Chuine 2000).

\subsection{Fitting parameter estimates}

To obtain a robust and biologically realistic model, we increased the amount of information during the parameterization of the model, following the approach used by Caffarra \& Eccel (2010). First, we used experimental information to restrict or fix possible parameter values to biologically realistic values. This was possible for the DORMPHOT model, more so, than for the UniForc or the Unified models, because its parameters could be related to previously observed responses. 
The experiments by Caffarra et al. (2011) showed that the critical photoperiod for dormancy induction $\left(D L_{\text {crit }}\right)$ in the IPG birch clone was $>12 \mathrm{~h}$ and $<14 \mathrm{~h}$. We thus fixed $D L_{\text {crit }}$ (indicating the photoperiod above which the rate of dormancy induction is low or zero) to $13 \mathrm{~h}$. Parameter $d F$ could be measured directly from experimental results and set to -0.174 (Caffarra et al. 2011). The range of values that could be adopted by the parameters $D_{\text {crit }}$ (critical state of dormancy induction), $C_{\text {crit }}$ (critical state of chilling) and $c C$ (upper threshold of chilling temperatures triggering a chilling rate of $\geq 0.5$ ) were restricted using the information available in the literature. $D_{\text {crit }}$ was bounded between 30 and 60, as dormancy induction in birch was reported to be triggered after 30-60 short days (ShDs) (Caffarra et al. 2011). $C_{\text {crit }}$ was bound between 30 and 80 , as birch dormancy was reported to be at least partially released after around 50 chilling days in experimental conditions (Caffarra et al. 2011) and before the beginning of January in field conditions (Skre et al. 2008, Murray et al. 1989). The parameter $c C$ was bound between 10 and 15, after Caffarra et al. (2011), and to the experimental studies on the subject, suggesting an upper threshold for active chilling temperature between $10^{\circ} \mathrm{C}$ (Sarvas 1974) and $12^{\circ} \mathrm{C}$ (Myking \& Heide 1995). As parameters $D L_{\text {crit }}$ and $d F$ were fixed before parameterisation, it was necessary to fit 9 parameters rather than 11.

Second, we used a varied phenological dataset, including data from different countries and latitudes (Table 1), to fit all parameters. The introduction of photoperiod made it necessary to fit the model on a dataset containing data from different latitudes, for a realistic adjustment of parameter values. The dataset used to fit the parameter estimates included the IPG birch dataset from Valentia (Ireland; $\mathrm{n}=24$ ) and data taken randomly from the IPG datasets for Bergen (Norway; $\mathrm{n}=6$ ) and for Freiburg and Wulfsdorf (Germany; $\mathrm{n}=6$ for both). The total number of years of the combined dataset was 42. Parameter estimates of the model are shown in Table 2.

The optimisation method employed for the calibration of the models was the Metropolis algorithm (Metropolis et al. 1953), which, in contrast to traditional numerical methods, such as the Downhill simplex method, allows for a more effective exploration of the parameter space in functions with many local 'minima', like phenological models (Chuine et al. 1998). This algorithm was repeated 20 times for all tested models (except the linear model, the parameters of which were determined by minimising a sum of squares function), in order to evaluate the consistency of parameter values.

Model fits were compared on the basis of model efficiency (ME) (Janssen \& Heuberger 1995) and using the corrected Akaike information criterion (AICc) (Burnham \& Anderson 2004) . The ME is defined as:

$$
\mathrm{ME}=\left(\mathrm{SS}_{\mathrm{tot}}-\mathrm{SS}_{\mathrm{res}}\right) / \mathrm{SS}_{\mathrm{tot}}
$$

where $\mathrm{SS}_{\text {tot }}$ is the sum of the squared deviations of the observations from their mean, and $\mathrm{SS}_{\text {res }}$ is the sum of squared residuals of the model fit. The AICc is defined as:

$$
\mathrm{AICC}=\mathrm{N} \times \ln \left(\frac{\mathrm{SS}_{\mathrm{res}}}{\mathrm{N}}\right)+2 k+\left(\frac{2 k(k+1)}{\mathrm{N}-k-1}\right)
$$

where $\mathrm{N}$ is the number of observations in the sample, $k$ is the number of parameters (e.g. 9 for DORMPHOT) and $\mathrm{SS}_{\text {res }}$ is the residual sum of squares. The ME quantifies the relative improvement in the prediction of budburst timing by the tested model over the 'benchmark' model, which is based on the average day of budburst. The AICc provides an estimate of model performance by taking into account both the model residuals and the number of fitted model parameters.

\subsection{Validation}

The models were validated on several datasets from the IPGs. Their sites of provenance were Johnstown Castle and JFK arboretum (Ireland, n = 24 and 10, re-

Table 2. Parameter estimates of each of the tested models. Par. range: parameter range (upper and lower values of the range). Parameter abbreviations, see Section 2

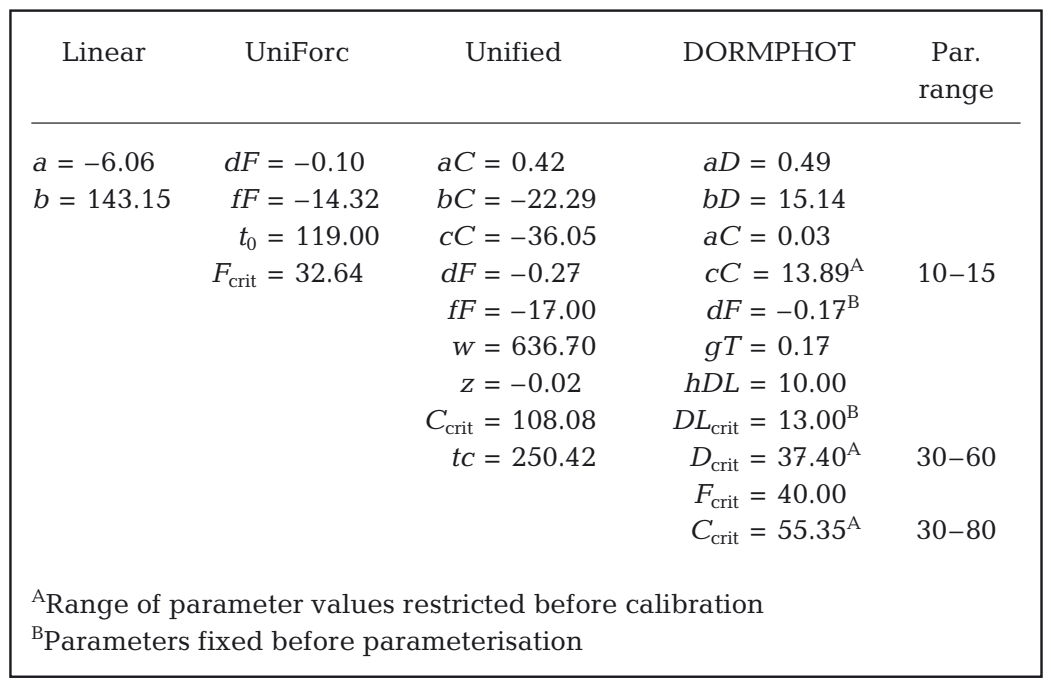


spectively); Bergen (Norway, $\mathrm{n}=7$ ); Freiburg, Munich and Wulfsdorf (Germany, $\mathrm{n}=26,17$ and 24, respectively); and Zurich (Switzerland, $\mathrm{n}=9$ ). Validation datasets were either totally independent of the calibration dataset or contained some of the observations used for model fit (semi-independent) (Table 1). For these semi-independent datasets indicators of model performance were also provided for those data that had not been used for calibration, for comparative purposes. Model performance was evaluated by ME and by mean absolute error (MAE). The MAE provides a mean measure of the model error, which does not allow for compensation between negative and positive errors (unlike the mean bias error, MBE, described below).

As one of the aims of the developed model was to forecast plant phenology in a warming climate, we evaluated the model accuracy in warm years with the 'warm year index' (WI), as described by Caffarra \& Eccel (2010). For each validation site, we selected the 3 years with the warmest February-April period, averaged the MAEs given by the tested models in those years, and compared them. The WI indicates model performance in warm years. Finally, model bias was evaluated by plotting observed versus predicted dates and by calculating the MBE (Mayer \& Butler 1993).

$$
\mathrm{MBE}=\frac{\left(\sum_{i=1}^{N} X_{\mathrm{pred}_{i}}-X_{\mathrm{obs}_{i}}\right)}{N}
$$

where $X_{\text {pred }}$ stands for the predicted dates, $X_{\text {obs }}$ for the observed dates and $N$ for the number of cases. A positive value of MBE indicates that, on average, the model overestimates actual observations; a negative value indicates that the model underestimates actual observations.

\section{RESULTS}

The Metropolis algorithm was successful at finding the 'best fit' set of parameter values. In fact, it yielded similar results at the end of each of its 20 repetitions.

All models performed well when describing the parameterisation dataset with a ME of $0.81,0.79,0.68$ and 0.71 and a MAE of 5.3, 5.7, 7.1 and 7.2 $\mathrm{d}$ for the DORMPHOT, Unified, UniForc and linear models, respectively. According to the AICc, which takes into account both the model residuals and the number of fitted model parameters, the DORMPHOT model was the best among all models tested (the AICs were 174.25, $177.85,179.64$ and 187.85 for the DORMPHOT, Unified, linear and UniForc models, respectively). The parameter estimates of the models are given in Table 2. The functions parameterised in the DORM-
PHOT model are shown in Fig. 2. Response functions showed a wide range of active dormancy induction temperatures (all temperatures below $\sim 20^{\circ} \mathrm{C}$, with optimal temperature $<10^{\circ} \mathrm{C}$, shown in Fig. 2a) and optimal dormancy induction photoperiods $<13 \mathrm{~h}$ (Fig. 2b). The fitted functions described a very wide range of active chilling temperatures (all temperatures below $\sim 15^{\circ} \mathrm{C}$, with optimal temperatures $<10^{\circ} \mathrm{C}$, shown in Fig. 2c) and an abrupt decrease in $D L_{50}$ upon exposure to 55 units of chilling $\left(C_{\text {crit }}\right)$ (Fig. 2d). On the other hand, the response to photoperiod was smoother: $T_{50}$ decreased slowly as photoperiod increased (Fig. 2e,f). The effect of chilling state and photoperiod on forcing rate clearly demonstrate that, for incompletely chilled plants, neither long days nor warm temperatures (or a combination of both) are able to trigger growth (Fig. 2g), while, for chilled plants, forcing rate increases with temperature and photoperiod (Fig. $2 \mathrm{~h}$ ).

The DORMPHOT model showed a good performance on all independent and semi-independent datasets, with the highest average $\mathrm{ME}$, lowest average MAE and lowest average WI (Table 3). The similar performance of the model on semi-independent datasets and independent datasets suggests that the model parameters were not site-biased. This is because the use of 6 yr from these datasets was unlikely to bias the set of parameter values obtained from a combined dataset containing 42 observations from 4 different stations.

While the DORMPHOT model showed the best average performance and a particularly good performance at Bergen compared to other models (MAE calculated on the entire dataset was 1.6, while the error for the only year not used in calibration was $+1.3 \mathrm{~d}$ ), the best performance on single datasets was sometimes yielded by other models. The Unified model yielded a slightly higher ME and lower MAE for Wulfsdorf (semi-independent dataset) and Munich. Similarly, the linear model yielded the lowest WI for JFK Arboretum (the DORMPHOT model yielded the second lowest), and the UniForc model yielded the lowest WI for Wulfsdorf, Freiburg and Munich (the DORMPHOT model yielded the second lowest). Dates of budburst were almost systematically overestimated for Wulfsdorf and Zurich (Fig. 3, MBE in Table 3) by all models, though less so by the DORMPHOT model.

\section{DISCUSSION}

The introduction of photoperiod into phenological models seems ecologically justified for Betula pubescens (birch), a species native to temperate and boreal zones with large fluctuations in daylength over the seasons. In these conditions, photoperiod provides a 

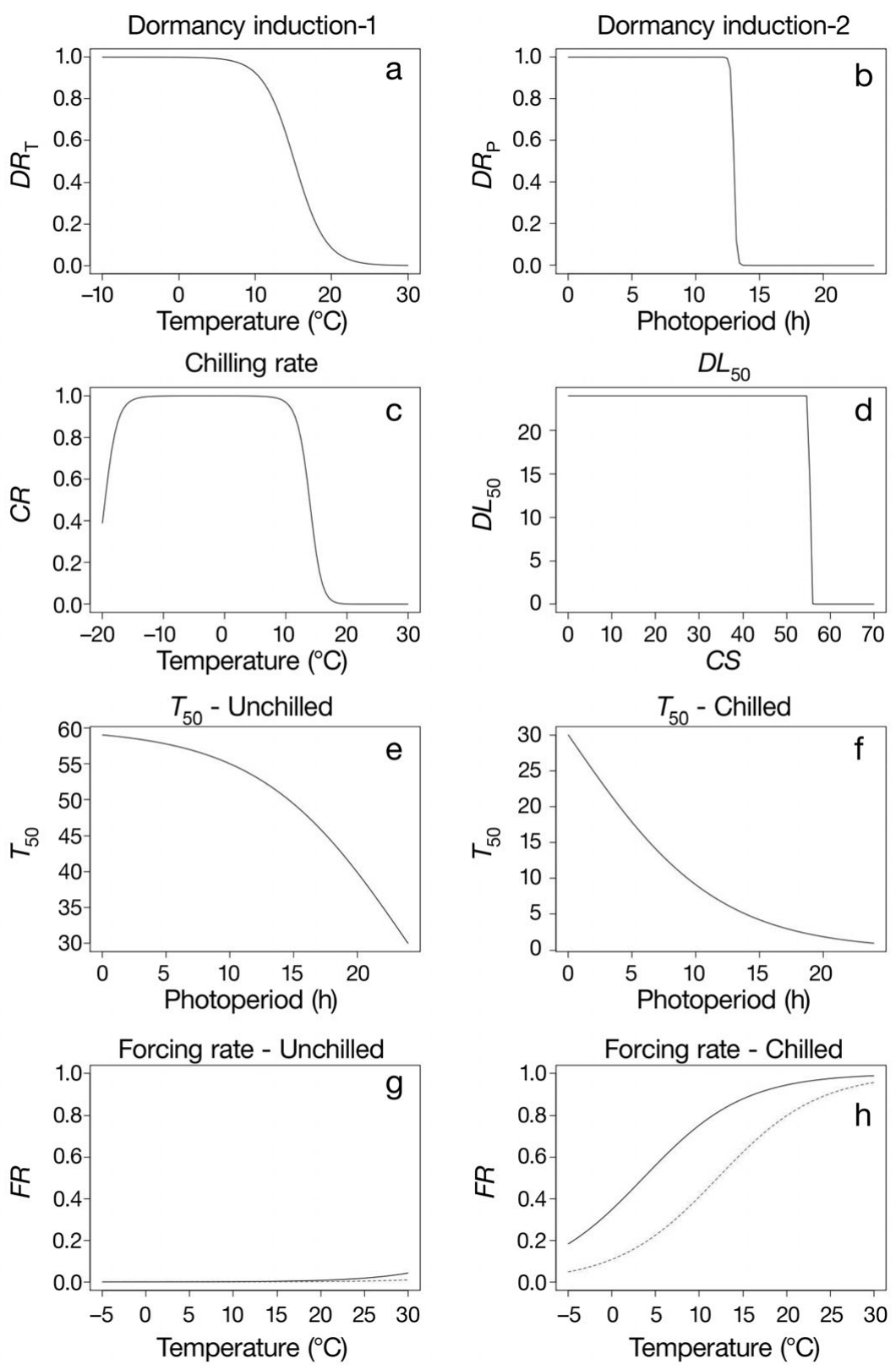

Fig. 2. Functions of the DORMPHOT model parameterised for Betula pubescens. (a) Temperature component of the rate of dormancy induction, $D R_{\mathrm{T}}$ function (Eq. 4); (b) photoperiod component of the rate of dormancy induction, $D R_{\mathrm{P}}$ function (Eq. 5); (c) chilling rate (Eq. 6); (d) $D L_{50}$ function (Eq. 9); (e) $T_{50}$ function for $C S=0$ (unchilled) (Eq. 8); (f) $T_{50}$ function for CS = 70 (chilled) (Eq. 8); (g) forcing rate for unchilled birch (Eq. 7); and (h) forcing rate for chilled birch (Eq. 7). Solid line: $D L=16 \mathrm{~h}$; dashed line: $D L=8 \mathrm{~h}$. Parameter abbreviations, see Section 2

reliable indicator of the time of the year and is thus exploited by trees to time their phenological transitions (Li et al. 2003). Its effects and interactions on the growth rate of birch buds have been reported by various authors (Myking \& Heide 1995, Heide 1993) and need to be taken into account if the model is to be ap- plied to data from different latitudes or to climate change scenarios. This effect of photoperiod could also prove to be important for other species in future climatic conditions.

Similarly, the introduction of dormancy induction (not previously considered in budburst models) was justified by the effect of late summer temperatures on the start of dormancy and subsequent phases, which has been shown in some studies. Heide (2003) showed that September temperature had a significant effect and explained as much as $20 \%$ of the variation in budburst date. Similarly, Caffarra et al. (2011) found that temperature during short-day dormancy induction significantly affected budburst timing, with high temperatures delaying the beginning of dormancy and subsequent budburst. If global temperature increases as predicted, more rapid bud growth in spring could be counterbalanced by later attainment of dormancy and later chilling fulfilment, which, in turn, could be balanced by a long photoperiod effect, with a resulting stabilising effect on budburst timing. Thus, the processes of dormancy and dormancy induction cannot be ignored if budburst models are to be applied to future climatic projections.

\subsection{Model fit}

Overfitting occurs when complex models are calibrated with a limited amount of data. The danger of overfitting is high for models of budburst considering dormancy, because the processes they describe are complex and the availability of large phenological datasets is often limited, due to the considerable length of time required to collect them (Linkosalo et al. 2008). This made it necessary to adopt some measures to avoid overfitting, following the method suggested by Caffarra \& Eccel (2010).

This approach, and the use of an appropriate model structure, was successful, as shown by the MAE yielded by the model fit on the calibration dataset (5.2), which was similar to the average MAE 
Table 3. Performance of the 4 models on the validation datasets. LIN: linear regression model between budburst date and the average temperature February-April; DP: DORMPHOT model; UF: UniForc model; UN: Unified model

\begin{tabular}{|c|c|c|c|c|c|c|c|c|c|c|c|c|c|c|c|c|}
\hline \multirow{2}{*}{$\begin{array}{l}\text { Site; no. of years } \\
\text { with observations }\end{array}$} & \multicolumn{4}{|c|}{ Modelling efficiency } & \multicolumn{4}{|c|}{ Mean absolute error } & \multicolumn{4}{|c|}{ Warm year index } & \multicolumn{4}{|c|}{ Mean bias error } \\
\hline & LIN & DP & UF & UN & LIN & DP & UF & UN & LIN & DP & UF & UN & LIN & DP & UF & UN \\
\hline Johnstown; $10^{\mathrm{a}}$ & 0.38 & 0.54 & 0.49 & 0.40 & 7.2 & 5.8 & 6.2 & 6.6 & 9.3 & 5.8 & 7.0 & 6.3 & 3.2 & -0.3 & 6.2 & 6.6 \\
\hline JFK Arboretum; $22^{\mathrm{a}}$ & 0.04 & 0.24 & -0.17 & -0.17 & 5.0 & 4.7 & 6.4 & 6.0 & 2.0 & 2.9 & 9.7 & 4.6 & 2.7 & 2.6 & 6.4 & 6.0 \\
\hline Wulfsdorf; $24^{\mathrm{b}}$ & 0.29 & 0.38 & 0.11 & 0.41 & 6.9 & 5.8 & 6.3 & 5.5 & 8.3 & 7.6 & 7.3 & 10.7 & 6.9 & 5.0 & 7.0 & 5.5 \\
\hline Wulfsdorf; 18 & 0.31 & 0.43 & 0.15 & 0.40 & 7.1 & 5.4 & 6.9 & 5.4 & 10.0 & 5.8 & 7.7 & 6.0 & 4.6 & 4.6 & 6.9 & 5.4 \\
\hline Freiburg; $26^{\mathrm{b}}$ & 0.03 & 0.67 & 0.58 & 0.67 & 8.4 & 4.6 & 5.0 & 4.5 & 8.2 & 4.1 & 3.0 & 7.6 & -6.4 & -1.3 & -1.5 & 4.5 \\
\hline Freiburg; 20 & -0.40 & 0.59 & 0.55 & 0.59 & 8.5 & 4.5 & 4.5 & 4.5 & 12.9 & 5.8 & 5.6 & 4.7 & -12.9 & -8.6 & -1.7 & -1.1 \\
\hline Munich; $17^{\mathrm{a}}$ & 0.34 & 0.63 & 0.53 & 0.70 & 6.8 & 4.7 & 5.2 & 4.1 & 8.0 & 4.3 & 4.0 & 4.7 & -0.9 & -1.3 & 1.4 & -2.3 \\
\hline Zurich; $9^{a}$ & 0.24 & 0.41 & -0.13 & 0.24 & 5.3 & 4.2 & 5.7 & 5.0 & 4.7 & 4.5 & 6.3 & 9.5 & 5.4 & 4.2 & 5.8 & 4.9 \\
\hline Bergen; $7^{\mathrm{b}}$ & 0.37 & 0.93 & 0.55 & 0.66 & 4.2 & 1.6 & 4.3 & 3.3 & 2.9 & 1.4 & 5.3 & 4.4 & 4.2 & -0.2 & -2.3 & -3.3 \\
\hline Mean (all) & 0.18 & 0.54 & 0.30 & 0.43 & 6.60 & 4.59 & 5.61 & 4.99 & 7.37 & 4.69 & 6.21 & 6.50 & 0.76 & 0.52 & 3.13 & 2.91 \\
\hline Mean (only ${ }^{a}$ ) & 0.15 & 0.47 & 0.24 & 0.36 & 6.65 & 4.88 & 5.82 & 5.27 & 7.82 & 4.85 & 6.72 & 6.00 & 0.35 & 0.20 & 4.17 & 3.25 \\
\hline
\end{tabular}
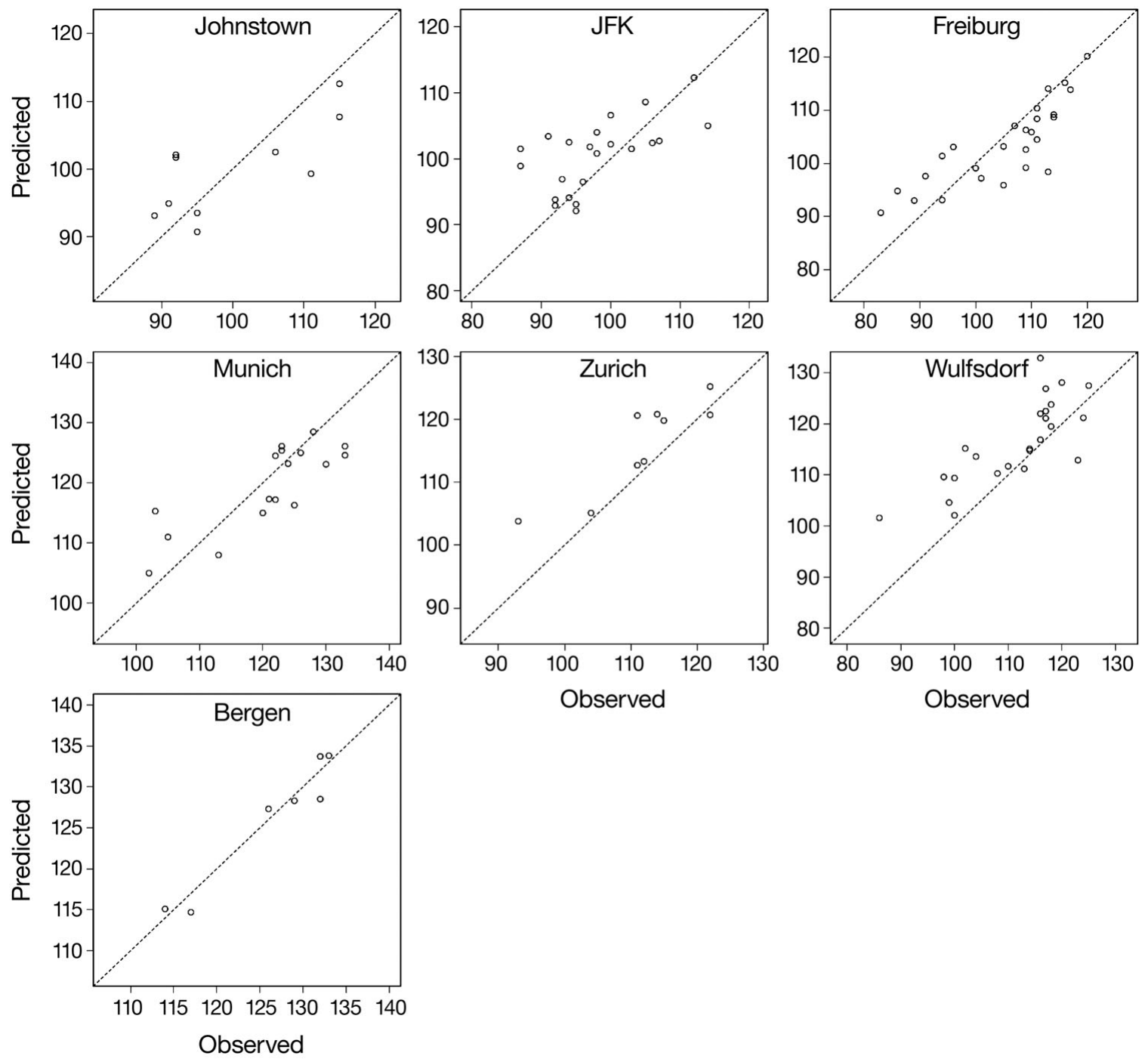

Fig. 3. Plots of observed versus predicted dates (day of the year) by the DORMPHOT model, for each validation dataset 
in external datasets (4.9). The AICc confirmed that the DORMPHOT was the model that best described the dataset among those tested. A notable feature of the DORMPHOT model was that each of its parameters could be related to an observed response or a directly or indirectly measurable rate. Usually, the empirical relationships fitted by phenological models are difficult or impossible to interpret in terms of actual phenological behaviour. In this case each parameter had a physiological meaning and could thus be fixed or restricted according to the information available.

Despite the constraints imposed during model calibration, in some cases, the fitted responses were unexpected. The chilling rate did not reflect the general theory that chilling temperatures in birch are active only $>0^{\circ} \mathrm{C}$ (Sarvas 1974, Myking \& Heide 1995), as it included a wide range of negative temperatures. At present, it is not possible to attribute this fitted response to the broad chilling sensitivity of this birch clone or to the lack of extreme temperatures in our calibration dataset that could bias the fitted parameters. Similarly, the sharp decrease in $D L_{50}$ around its inflection point, $C_{\text {crit }}$, was unexpected. As the $D L_{50}$ curve expresses the dynamic transition from the dormant (non-reactive) to the non-dormant (reactive to temperature and photoperiod) state, the abrupt decrease in $D L_{50}$ mimicked an abrupt dormancy release, without any partially chilled stage in between. Yet, the experiments conducted by Myking \& Heide (1995) and Heide (1993) have shown an intermediate behaviour in partially chilled plants, with growth occurring at a lower rate. Fitting a realistic response to chilling temperatures remains a difficult task, as noted in previous studies (Linkosalo et al. 2008, Garcia de Cortazar Atauri et al. 2009), since dormancy release is difficult to measure and very few observations of this phenophase exist. However, phenological models have to take the dormancy phase into account in order to ensure accurate predictions of budburst dates for the future. Correct parameterisation of the dormancy phase could be obtained by using phenological data taken in extreme conditions (cold and warm), but, as long as winter chilling conditions are not limiting for trees in their current geographical range, calibrating the dormancy phase on field data will be difficult.

Given these shortcomings, the functions fitted in the DORMPHOT model were in general agreement with the observations made during controlled environment experiments. The model prediction error was minimised at the centre of the parameter space that we defined using our experimental observations, rather than assuming values at its extreme (see Table 2); this confirms the biological realism of the model structure and its agreement with the observed processes. The temperature-dependent component of dormancy induction (Fig. 2a) is in accordance with experimental observations of a faster dormancy induction at $10^{\circ} \mathrm{C}$ (Li et al. 2003, Caffarra et al. 2011). The model also predicted that forcing rate (and thus budburst rate) was zero for unchilled birch regardless of photoperiod, but increased with temperature and photoperiod in chilled birch, as experimentally observed by Caffarra et al. (2011) and by Myking \& Heide (1995). Finally, the critical thresholds $\left(D_{\text {crit, }} C_{\text {crit }}\right.$ and $\left.F_{\text {crit }}\right)$ fitted in the model agreed with the experimental observations of Caffarra et al. (2011). The model predicts dormancy establishment upon exposure to ShDs and cold temperature $\left(<10^{\circ} \mathrm{C}\right)$ for $38 \mathrm{~d}$, dormancy release upon exposure to temperatures $<10^{\circ} \mathrm{C}$ for $55 \mathrm{~d}$, and subsequent budburst upon exposure to LDs and temperatures $>20^{\circ} \mathrm{C}$ for $40 \mathrm{~d}$. Caffarra et al. (2011) observed that dormancy was induced by 30 to 60 ShDs at $10^{\circ} \mathrm{C}$, after which between 50 and $80 \mathrm{~d}$ at $10^{\circ} \mathrm{C}$ were able to trigger dormancy release, and mean times to budburst at $20^{\circ} \mathrm{C}$ for minimally chilled birch (50\% budburst) were about $30 \mathrm{~d}$.

\subsection{Model validation}

The high average modelling efficiency and better performance of the DORMPHOT model on validation datasets was notable, given the presence of additional relationships constraining the model structure and the restriction of parameter space during calibration. This suggests that the model structure was suited to the shape of the data and the noise in the series did not affect the parameter values. The consistently more accurate budburst prediction output by the DORMPHOT model in warm years and the similarity between its average MAE and WI suggests that it is robust for extreme years and can be reliably applied to future climate scenarios. Whereas the model, in general, tended to provide unbiased predictions, it markedly overpredicted actual observations for Wulfsdorf and Zurich. The overpredicted phenology for these 2 sites might result from a systematic bias in temperature records or in the observations of budburst. It could also be due to micro-climatic differences between the IPG sites and the weather stations ( 2 and $16 \mathrm{~km}$ away for Wulfsdorf and Zurich, respectively). Alternatively, it could result from some environmental effect unaccounted for by the model, or a locally adapted phenological pattern (i.e. acclimation). Whereas the IPG birches have all been cloned from the same individual, even genetically identical individuals can sometimes display contrasting traits (Veitia 2005). 


\section{CONCLUSIONS}

Whereas the DORMPHOT model is built upon existing phenological models, it features 2 novel aspects: (1) the inclusion of dormancy induction through the combined effect of temperature and photoperiod and (2) the dynamic effect of photoperiod on forcing rate. Besides giving more biological realism to the model, these modifications improved its predictive performance. The model outperformed the Unified model, the linear regression model and the UniForc model. However, it is not clear whether this improvement is to be attributed to both the newly introduced mechanisms (dormancy induction and photoperiod sensitivity during forcing) or to just one of them. To answer this question, sensitivity analysis to identify which model components exert the most influence on model results should be performed in a further study.

Also, further refinement of the model could be obtained by the use of an even wider range of phenological data for a better adjustment of parameter values. In any case, the DORMPHOT model showed a notable robustness, which was consistent in warm years and over a wide climatic and latitudinal range. These features support its application to climate change scenarios for the forecast of its impacts on tree phenology.

Acknowledgements. The authors thank Fabio Zottele for advice and proofreading and acknowledge the support of the Irish Environmental Protection Agency under the Project Climate Change Impacts on Phenology; implications for terrestrial ecosystems (STRIVE programme, Project Number 2007-CCRP-2.4), and of the European Union under COST action 725

\section{LITERATURE CITED}

Battey N (2000) Aspects of seasonality. J Exp Bot 51:1769-1780

Burnham KP, Anderson DR (2004) Multimodel inference: understanding AIC and BIC in model selection. Sociol Methods Res 33:261-304

> Caffarra A, Eccel E (2010) Increasing the robustness of phenological models for Vitis vinifera cv. Chardonnay. Int $\mathrm{J}$ Biometeorol 54:255-267

Caffarra A, Donnelly A, Chuine I, Jones MB (2011) Modelling the timing of Betula pubescens budburst. I. Temperature and photoperiod: a conceptual model. Clim Res 46:147-157

Campbell RK, Sugano AI (1975) Phenology of bud burst in Douglas-fir related to provenance, photoperiod, chilling, and flushing temperature. Bot Gaz 136:290-298

Cannell MGR, Smith RI (1983) Thermal time, chill days and prediction of budburst in Picea sitchensis. J Appl Ecol 20: 951-963

Chmielewski FM (1996) The international phenological gardens across Europe. Present state and perspectives. Phenology and Seasonality 1:19-23

Chuine I (2000) A unified model for budburst of trees. J Theor Biol 207:337-347

Chuine I, Cour P, Rousseau DD (1998) Fitting models predict- ing dates of flowering of temperate-zone trees using simulated annealing. Plant Cell Environ 21:455-466

Donnelly A, Salamin N, Jones MB (2006) Changes in tree phenology: an indicator of spring warming in Ireland? Biol Environ Proc R Ir Acad 106:47-55

Garcia de Cortazar-Atauri I, Brisson N, Gaudillere JP (2009) Performance of several models for predicting budburst date of grapevine (Vitis vinifera). Int J Biometeorol 53: $317-326$

Håbjørg A (1972) Effects of photoperiod and temperature on growth and development of three latitudinal populations of Betula pubescens Ehrh. Melding Norges Landbrukshøgskole 51:1-27

> Häkkinen R, Linkosalo T, Hari P (1998) Effects of dormancy and environmental factors on timing of bud burst in Betula pendula. Tree Physiol 18:707-712

Hänninen H (1990) Modelling dormancy release in trees from cool and temperate regions. Acta For Fenn 213:34-43

Hänninen H (1995) Effects of climatic change on trees from cool and temperate regions: an ecophysiological approach to modelling of bud burst phenology. Can J Bot 73: 183-199

> Heide OM (1993) Daylength and thermal time responses of budburst during dormancy release in some northern deciduous trees. Physiol Plant 88:531-540

Heide OM (2003) High autumn temperature delays spring budburst in boreal trees counterbalancing the effect of climate warming. Tree Physiol 23:931-936

> Hunter AF, Lechowicz MJ (1992) Predicting the timing of budburst in temperate trees. J Appl Ecol 29:597-604

Janssen PHM, Heuberger PSC (1995) Calibration of processoriented models. Ecol Modell 83:55-66

Kellomaki S, Kolstrom M (1994) The influence of climatechange on the productivity of Scots pine, Norway spruce, pendula birch and pubescent birch in southern and northern Finland. For Ecol Manag 65:201-217

Kramer K (1994) Selecting a model to predict the onset of growth of Fagus sylvatica. J Appl Ecol 31:172-181

- Kramer K, Leinonen I, Loustau D (2000) The importance of phenology for the evaluation of impact of climate change on growth of boreal, temperate and Mediterranean forest ecosystems: an overview. Int J Biometeorol 44:67-75

Landsberg JJ (1974) Apple fruit bud development and growth; analysis and an empirical model. Ann Bot (Lond) 38:1013-1023

Leinonen I, Kramer K (2002) Applications of phenological models to predict the future carbon sequestration potential of boreal forests. Clim Change 55:99-113

Li C, Junttila O, Ernstsen A, Heino P, Palva ET (2003) Photoperiodic control of growth, cold acclimation and dormancy development in silver birch (Betula pendula) ecotypes. Physiol Plant 117:206-212

Lieth $\mathrm{H}$ (1997) Aims and methods in phenological monitoring. In: Lieth $\mathrm{H}$, Schwartz MD (eds) Phenology in seasonal climates. I. Backhuys Publisher, Leiden

Linkosalo T, Lappalainen H, Hari P (2008) A comparison of phenological models of leaf budburst and flowering of boreal trees using independent datasets. Tree Physiol 28: 1873-1882

Mayer DG, Butler DG (1993) Statistical validation. Ecol Modell 68:21-22

Metropolis N, Rosenbluth AW, Rosenbluth MN, Teller AH (1953) Equation of state calculations by fast computing machines. J Chem Phys 21:1087-1093

Murray MB, Cannell MGR, Smith RI (1989) Date of budburst of fifteen species in Britain following climatic warming. J Appl Ecol 26:693-700 
Myking T, Heide OM (1995) Dormancy release and chilling requirement of buds of latitudinal ecotypes of Betula pendula and Betula pubescens. Tree Physiol 15:697-704

Piao S, Friedlingstein P, Ciais P, Viovy N, Demarty J (2007) Growing season extension and its impacts on terrestrial carbon cycle in the Northern Hemisphere over the past 2 decades. Global Biogeochem Cycles 21:GB3018-1-11. doi:10.1029/2006GB002888

Pop EW, Oberbauer SF, Starr G (2000) Predicting vegetative bud break in two arctic deciduous shrub species, Salix pulchra and Betula nana. Oecologia 124:176-184

Richardson EA, Seeley SD, Walker DR (1974) A model for estimating the completion of rest for 'Redhaven' and 'Elberta' peach trees. HortScience 9:331-332

Sarvas R (1972) Investigations on the annual cycle of development of forest trees. Active period. Commun Inst For Fenn

Editorial responsibility: Tim Sparks,

Cambridge, UK
$76: 1-110$

Sarvas R (1974) Investigations on the annual cycle of development of forest trees. II. Autumn dormancy and winter dormancy. Commun Inst For Fenn 84:1-101

Skre O, Taulavuori K, Taulavuori E, Nilsen J, Igeland B, Laine K (2008) The importance of hardening and winter temperature for growth in mountain birch populations. Environ Exp Bot 62:254-266

Thomas B, Vince-Prue D (1997) Photoperiodism in plants. Academic Press, London

Veitia RA (2005) Stochasticity or the fatal imperfection of cloning. J Biosci 30:21-30

Welling A, Kaikuranta P, Rinne P (1997) Photoperiodic induction of dormancy and freezing tolerance in Betula pubescens. Involvement of $\mathrm{ABA}$ and dehydrins. Physiol Plant 100:119-125

Submitted: April 3, 2010; Accepted: December 17, 2010

Proofs received from author(s): February 23, 2011 